\title{
Neuroprotective effects of urate are mediated by augmenting astrocytic glutathione
}

synthesis and release.

Rachit Bakshi ${ }^{* 1}$, Hong Zhang ${ }^{1,2}$, Robert Logan ${ }^{1}$, Ila Joshi ${ }^{3}$, Yuehang $\mathrm{Xu}^{1}$, Xiqun $\mathrm{Chen}^{1}$ and Michael A. Schwarzschild ${ }^{1}$.

${ }^{1}$ Department of Neurology, Massachusetts General Hospital, Boston, MA 02129

${ }^{2}$ Department of Neurobiology, Key Laboratory for Neurodegenerative Disease of the Ministry of Education, Capital Medical University, Beijing 100069, China

${ }^{3}$ Department of Dermatology, Massachusetts General Hospital, Boston, MA 02129

\section{${ }^{*}$ Corresponding author:}

Rachit Bakshi, PhD

Molecular Neurobiology Laboratory,

MassGeneral Institute for Neurodegenerative Disease

Massachusetts General Hospital

$11416^{\text {th }}$ street

Boston, MA 02129

Email:rbakshi1@mgh.harvard.edu

Phone: +1617 7242575

Fax: +1617724 1480

\section{Word Count}

- Abstract: 167

- Total word count: 4875

- Total Figures: 4

- Tables: 0 


\begin{abstract}
Urate has emerged as a promising target for neuroprotection based on epidemiological observations, preclinical models, and early clinical trial results in multiple neurologic diseases, including Parkinson's disease (PD). This study investigates the astrocytic mechanism of urate's neuroprotective effect. Targeted biochemical screens of conditioned medium from urate- versus vehicle-treated astrocytes identified markedly elevated glutathione (GSH) concentrations as a candidate mediator of urate's astrocyte-dependent neuroprotective effects. Urate treatment also induced the nuclear translocation of the nuclear factor (erythroid-derived 2)-like 2 (Nrf2) protein and transcriptional activation of its key target genes in primary astrocytic cultures. Urate's neuroprotective effect was attenuated when GSH was depleted in the conditioned media either by targeting its synthesis or release by astrocytes. Overall, these results implicate GSH as the extracellular astrocytic factor mediating the protective effect of urate in a cellular model of PD. These results also show that urate can employ a novel indirect neuroprotective mechanism via induction of the Nrf2 signaling pathway, a master regulator of the response to oxidative stress, in astrocytes.
\end{abstract}

\title{
Keywords
}

- Astrocytes\Glutathione\Neurons\Nrf2\Urate\} 


\section{Introduction}

Urate (2,6,8-trioxy-purine; a.k.a. uric acid) has been gaining momentum as a promising candidate therapeutic target for people with Parkinson's disease (PD) based on its antioxidant and neuroprotective properties, and on its identification as a predictor of a reduced PD risk and a favorable rate of disease progression (Cipriani et al., 2010). Several groups including ours have documented protective properties of urate in cellular and rodent models of PD and other neurodegenerative diseases (Bakshi et al., 2015). In dopaminergic cell lines, urate blocked cell death and oxidative stress induced by 6-hydroxydopamine (6-OHDA), dopamine or rotenone (Zhu et al., 2012; Jones et al., 2000; Duan et al., 2002). Urate at physiologically relevant concentrations enhanced function and survival of dopaminergic neurons in primary cultures of rat ventral mesencephalon (Guerreiro et al., 2009). Urate also confers protection in various cellular models of neurotoxicity beyond that of PD. Urate protected cultured spinal cord or hippocampal neurons from excitotoxic (glutamate-induced) (Yu et al., 1998; Du et al., 2007) or nitrosative (peroxynitrite-induced) cell death (Scott et al., 2005). Similarly, in intact animals urate protects these neurons from ischemic brain or compressive spinal cord injuries (Yu et al., 1998; Scott et al., 2005).

The neuroprotective effects of urate have also been evaluated in vivo in rodent models of PD, and was found to attenuate 6-OHDA toxicity (Gong et al., 2012). Similarly, our group found that mice with a urate oxidase gene (UOx) knockout have elevated brain urate levels and are resistant to toxic effects of 6-OHDA on nigral dopaminergic cell counts, striatal dopamine content, and rotational behavior (Chen et al., 2013). Conversely, transgenic over-expression of UOx exacerbated these anatomical, neurochemical, and behavioral deficits of the lesioned dopaminergic nigrostriatal pathway.

Although considerable evidence indicates that urate is a powerful direct antioxidant few studies have investigated alternative mechanisms of its protective effect. Previous findings of our group (Cipriani et al., 2012a) and others (Du et al., 2007) have suggested a prominent role of astrocytes in the neuroprotective effects of urate. We demonstrated an essential requirement for astrocytes in order for urate to fully protect dopaminergic cells (Cipriani et al., 2012a) or nigral neurons (Cipriani et al., 2012b). We further determined that in response to urate, astrocytes release a potent neuroprotective factor, which differs from urate because it is insensitive to urate-eliminating incubation with commercial UOx enzyme. In the present study we identify and characterize the astrocytic protective factor and signaling pathways mediating urate's neuroprotective effect on dopaminergic cells. 


\section{Materials and Methods}

\section{Cell Cultures, Drug Treatment and Conditioned Media Experiments}

Astroglial cultures were prepared from the cerebral cortex of 1- or 2- day-old neonatal mice as described previously (Cipriani et al., 2012a). Our astroglial cultures comprised $>95 \%$ astrocytes, $<2 \%$ microglial cells, $<1 \%$ oligodendrocytes, and no detectable neuronal cells. Astrocyte cultures reached confluence after 7-10 days in vitro. Urate was dissolved in DMEM as $20 \mathrm{X}$ concentrated stocks. Enriched astroglial cultures were treated with $100 \mu \mathrm{M}$ urate or vehicle. Twenty-four hours later conditioned media (CM) were collected and filtered through a $0.2 \mu \mathrm{m}$ membrane to remove cellular debris and immediately used for experiments. All reagents for cell culture were obtained from Life Technologies.

The rodent MES 23.5 dopaminergic cell line, which was derived from the fusion of a dopaminergic neuroblastoma and embryonic mesencephalon cells (Crawford et al., 1992), was obtained from Dr. Weidong Le at Baylor College of Medicine (Houston, USA). The cells were cultured as described previously (Cipriani et al., 2012a). MES 23.5 cells were incubated with $\mathrm{CM}$ from urate- or vehicle-treated astrocytes for $24 \mathrm{~h}$ before addition of $200 \mu \mathrm{M} \mathrm{H}_{2} \mathrm{O}_{2}$ and incubation for another $24 \mathrm{~h}$.

\section{RNA Isolation and Quantitative Real-Time PCR}

RNA was extracted from astrocyte cultures by TRIzol (GIBCO/BRL) extraction. RNA quality was determined by spectrophotometry and by visual inspection of electropherograms using the RNA 6000 NanoChip Kit on the Agilent 2100 Bioanalyzer (Agilent Technologies). cDNA synthesis was performed using Superscript ${ }^{@}$ VILO cDNA synthesis kit (Invitrogen). For quantitative gene expression analysis, SYBR green primers and probes (Applied Biosystems) were used. The specificity of each PCR product was confirmed by melting dissociation curve $\left(\mathrm{T}_{\mathrm{m}}\right)$ analysis. The comparative threshold cycle method was used for quantitative analysis. Glyceraldehyde-3-phosphate dehydrogenase (GAPDH) and RPL13 ribosomal RNA were used as RNA loading controls. Equal amplification efficiencies were confirmed for target and reference genes. Primer pairs used for quantitative PCR are listed as follows: mouse GCLM (forward 5'-GCA CAG GTA AAA CCC AAT AG-3', reverse 5'-TTA GCA AAG GCA GTC AAA TC-3'), mouse GCLC (5'-CTA TCT GCC CAA TTG TTA TGG-3', reverse 5'ACAGGTAGCTATCTATTGAGTC-3'), mouse NQO1 (forward 5'-CCT TTC CAG AAT AAG AAG ACC-3'), reverse 5'-AAT GCT GTA AAC CAG TTG AG-3') and mouse HO-1 (forward 5'- CAA CCC CAC CAA GTT CAA ACA-3', reverse 5'- AGG CGG TCT TAG CCT CTT CTG-3'). 


\section{Western Blot Analysis}

Western blot analysis was performed using lysates from astrocytes. The blots were probed with antibodies against NQO1 (Sigma N5288), GCLC (Abcam ab53179), GCLM (Abcam ab126704) and $\beta$-actin (Abcam ab8227) using the dilutions recommended in the product datasheet. Band densities were analyzed with ImageJ software.

\section{Immunofluorescence Microscopy}

Primary astrocytes cultures grown on chamber slides were fixed in $4 \%$ paraformaldehyde for $20 \mathrm{~min}$ at room temperature and then permeabilized for $30 \mathrm{~min}$ with $0.1 \%$ Tween-20 in phosphate-buffered saline (PBS). After blocking the cells in $3 \%$ bovine serum albumin/PBS for $1 \mathrm{~h}$, anti-Nrf2 and anti-glial fibrillary acidic protein (GFAP) antibodies were added at $4^{\circ} \mathrm{C}$ overnight, followed by incubation with Cy3-conjugated secondary antibody for GFAP and fluorescein isothiocyanate (FITC) conjugated secondary antibody for Nrf2 (Molecular Probes) for another $1 \mathrm{~h}$. Slides were treated with VECTASHIELD mounting medium containing DAPI (4',6-diamidino-2-phenylindole; Vector Laboratories) and mounted. Images were collected with a Nikon $A 1+/ A 1 R+$ confocal microscope and were processed with NIS Element confocal imaging software.

Fluorescence intensity of Nrf2 in the nuclear and cytoplasmic regions was quantified using ImageJ (http://rsbweb.nih.gov/ij/) as described (Drake et al., 2010). Background-corrected nuclear:cytoplasmic (N/C) ratios were calculated from mean fluorescence intensities measured within a small circular region of interest placed randomly in a region of uniform staining devoid of any punctate structures within the nucleus and the cytoplasm of each cell.

\section{Cell Viability Assays}

For evaluation of cell viability, cells were co-stained with propidium iodide ( $\mathrm{PI})$ / annexin V and analyzed using fluorescence activated cell sorting (FACS) as described before (Behbahani et al., 2005). The percentage of apoptotic cells was determined by FACScan Flow Cytometer and CellQuest software (Becton Dickinson).

\section{Screening for Neurotrophic Factors}

Screening for GDNF, BDNF and IL6 was conducted using commercial ELISA kits as per manufacturer (Abcam) instructions. Total GSH levels (GSH+GSSG) were measured in lysates and CM from urate- and vehicle-treated astrocytes. Levels of GSH were determined by a simple 
in vitro fluorometric detection assay kit as per manufacturer (Abcam ab65322) instructions. All GSH measurements were normalized to total protein levels.

\section{GSH Depletion Assays}

MK-571 and BSO were purchased from Sigma and dissolved in DMEM as 100X concentrated stocks. Enriched astroglial cultures were treated with vehicle or urate $(100 \mu \mathrm{M})$ alone or in combination with BSO $(0.25 \mathrm{mM})$ or MK-571 $(50 \mu \mathrm{M})$. Twenty-four hours later CM was collected and filtered through a $0.2 \mu \mathrm{m}$ membrane to remove cellular debris and immediately used for experiments with MES 23.5 cells. To verify the effects of BSO or MK-571, GSH content in the CM was measured. MES 23.5 cells were pretreated with vehicle CM or urate $\mathrm{CM}$, with or without BSO or MK-571, (or were not pretreated) before being exposed to oxidative stressor $\left(\mathrm{H}_{2} \mathrm{O}_{2}\right)$ for $24 \mathrm{~h}$. The percentage of dead cells was analyzed using the FACS method described above.

\section{Statistical Analysis}

Values were expressed as mean \pm standard error of the mean (SEM). Differences between groups were examined for statistical significance using one-way ANOVA or two-tailed Student's t-tests, using GraphPad Prism 5 software. A p value less than 0.05 denoted the presence of a statistically significant difference. 


\section{Results}

\section{Urate induces GSH release from cultured primary astrocytes}

First we confirmed the neuroprotective effect of conditioned medium (CM) from uratetreated astrocytes by assessing dopaminergic cell death with a method complementary to those of our previous report (Cipriani et al 2012a). Using a Pl/annexin dual staining method to evaluate cell viability we observed complete protection of dopaminergic MES 23.5 cells from oxidative stressor $\left(\mathrm{H}_{2} \mathrm{O}_{2}\right)$-induced cell death after their incubation with $\mathrm{CM}$ from astrocytes treated with $100 \mu \mathrm{M}$ urate (Fig. 1A).

To identify putative inducible factors secreted by urate-treated astrocytes we conducted a targeted screen of their CM for prominent neurotrophic factors known to be released by astrocytes. Using commercial assays we measured levels of glial cell line-derived neurotrophic factor (GDNF), brain-derived neurotrophic factor (BDNF), interleukin-6 (IL6) and GSH in urateand vehicle-treated glial CM. No significant difference was found in the levels of BDNF, IL6 and GDNF in urate- versus vehicle-treated CM (Fig. 1B). In contrast, GSH levels were significantly higher in $\mathrm{CM}$ from urate- compared to vehicle-treated astrocytes.

\section{Urate induces GSH levels and Nrf2-targeted gene in astrocytes}

To determine the mechanism of increased extracellular GSH levels after urate treatment, we examined changes in GSH levels as well as GSH synthesis within primary astrocytes. Urate treatment for $24 \mathrm{~h}$ significantly increased GSH levels in the astrocytes compared to those in control astrocytes (Fig. 2A). We also examined the protein and transcript levels of two subunits of the Y-glutamyl cysteine ligase (GCL), the rate-limiting enzyme of GSH synthesis. Both the protein and mRNA expression levels of the modifier subunit (GCLM) were significantly induced by urate treatment (Fig $2 \mathrm{~B}$ and $2 \mathrm{C}$ ). Because $\mathrm{GCL}$ is transcriptionally regulated by nuclear factor (erythroid-derived 2)-like 2 (Nrf2), we also measured the mRNA and protein expression of other key Nrf2-regulated genes including $\mathrm{NAD}(\mathrm{P}) \mathrm{H}$ :quinone oxidoreductase 1 (NQO1) and heme oxygenase-1 (HO-1). Urate treatment for $24 \mathrm{~h}$ led to a significant increase in mRNA levels of NQO1 in astrocyte cultures (Fig. 2C) and a trend towards an increase in its protein levels (Fig 2B). Transcript levels of HO-1 also appeared increased, though not significantly, with urate treatment (Fig. 2C). The protein levels of HO-1 were not detected by the western blot analysis. 


\section{Urate induces nuclear localization of Nrf2 in astrocytes}

Activation of the Nrf2 pathway involves translocation of the Nrf2 protein from the cytoplasm into the nucleus where it can transactivate its targets. Nrf2 is known to bind to the antioxidant-response element (ARE) in promoter regions of Nrf2-responsive genes leading to their transcriptional activation in response to oxidative stress or related external stimuli. Here, the subcellular distribution of Nrf2 was studied by immunofluorescence confocal microscopy. We observed significant redistribution of Nrf2 immunoreactivity from its relatively balanced cytoplasmic and nuclear localization in vehicle-treated astrocytes to a predominantly nuclear localization after treatment with urate (Fig. 3). Cultures were also stained with DAPI to visualize nuclei and immunostained with astrocyte-specific anti-GFAP antibody.

\section{GSH depletion attenuates neuroprotection by conditioned medium from urate-treated astrocytes}

To determine whether GSH mediates urate's neuroprotective effect we reduced the GSH concentration of CM from urate-treated astrocytes. This was accomplished by two strategies. First, GSH synthesis in urate-treated astrocytes was inhibited by buthionine sulfoximine (BSO), which inhibits GCL, the rate-limiting enzyme of GSH synthesis (Drew et al., 1984). Second, we blocked GSH release from the astrocytes by inhibiting multidrug resistance protein 1 (MRP1) transporter with its competitive inhibitor MK-571 (Hirrlinger et al., 2002). We performed cell viability experiments in MES 23.5 dopaminergic cells treated with CM from astrocytes treated with vehicle or urate in combination with BSO $(0.25 \mathrm{mM})$ or MK-571 $(50 \mu \mathrm{M})$. These drugs were added to the astrocytes concurrently with urate or vehicle $24 \mathrm{~h}$ prior to the collection of the CM. GSH levels were undetectable in urate or vehicle CM from astrocytes treated with either BSO or MK-571 (Fig. 4A). There was no significant effect of BSO or MK-571 treatment alone on MES 23.5 cell viability in absence of $\mathrm{H}_{2} \mathrm{O}_{2}$ (Fig. 4B and 4C). Neuroprotection by CM from uratetreated astrocytes was significantly reduced by BSO or MK-571 treatment as depicted by increased cell death compared to urate $\mathrm{CM}$ alone (Fig. 4B and $4 \mathrm{C}$ ), indicating that the presence of GSH in the CM is critical for urate's protective effect. 


\section{Discussion}

Its well-documented direct antioxidant properties notwithstanding, urate can produce much of its neuroprotective effect indirectly via astrocytes (Cipriani et al., 2012a; Du et al., 2007). They in turn release a potent neuroprotective factor, which differs from urate because incubation of medium conditioned by urate-treated astrocytes with commercially obtained UOX eliminates urate but not the protective influence (Cipriani et al 2012a). Here we identified GSH as a primary candidate for the putative neuroprotective factor that is released from urate-treated astrocytes based on its markedly higher concentration in CM and lysates from urate-treated compared to control astrocytes. The glutathione system is very important for cellular defense and protects against a variety of different reactive oxygen species (ROS). The total GSH content of astroglial cultures measured in the astrocytes and the extracellular media was in the same physiological range as reported previously (Raps et al., 1989, Dringen et al 1999). GSH is a tripeptide that is synthesized by two successive enzymatic reactions. The first, rate-limiting step in GSH biosynthesis is catalyzed by GCL $(\mathrm{Lu}, 2013)$. In its catalytically most active form, GCL comprises a catalytic subunit (GCLC) and a modifier subunit (GCLM). Urate significantly increased the transcript and protein levels of the modifier (but not the catalytic) subunit of the enzyme in astrocytes, likely contributing to their increased GSH synthesis given that GCLM increases $V_{\max }$ of GCLC and its affinity for its substrates (Franklin et al., 2009). Although the significance of differential induction of GCLM versus GCLC genes is unclear, it is consistent with similarly discordant GCL subunit regulation by other extracellular stimuli (Cai et al., 1995; Cai et al., 1997; Franklin et al., 2009; Moellering et al., 1998).

The GCL genes are part of a broader cellular antioxidant pathway that controls a set of effector genes through a unique cis-acting transcriptional regulatory sequence, termed the antioxidant response element (ARE). Several lines of evidence suggest that Nrf2 is a transcription factor responsible for upregulating ARE-mediated gene expression (Kensler et al., 2007). The Nrf2 pathway has been known to be activated by both oxidative stress as well as antioxidants (Ma, 2013). From our findings, urate appears to be a key activator of Nrf2 signaling and its downstream targets that guard against oxidative stress. Astrocytes are known to interact with surrounding neurons and their neuroprotective properties are well documented (Maragakis et al 2006, Bélanger et al 2009, Sidoryk-Wegrzynowicz et al 2011). Here we demonstrate an unanticipated requirement for a neuroprotective factor released from astrocytes in order for urate to fully protect dopaminergic cells in a cellular model of PD. Interestingly, others have recently reported Nrf2 involvement in urate's neuroprotective effects on dopaminergic cells 
(Zhang et al., 2014). They reported that urate protected dopaminergic cell lines in the absence of glial cells but, as noted by the authors, at much ( 100-fold) higher concentrations of urate than are needed in the presence of astrocytes (Cipriani et al .,2012a; Zhang et al 2014). They found urate to be protective at concentrations of $200 \mu \mathrm{M}$ and above, whereas CNS concentrations of endogenous urate are typically 10- to 100-fold lower (Ascherio et al., 2009; Chen et al., 2013). Thus activation of astrocytic Nrf2 signaling by urate and its indirect neuroprotective effects may be more pathophysiologically relevant than are the direct effects of urate on neuronal cells. The importance of astrocytic Nrf2 is in agreement with earlier demonstrations that Nrf2 induction in astrocytes boosts their production of GSH, which in turn can protect glial cells and neighboring neurons (Kraft et al., 2004; Shih et al., 2003). The protective effects of astrocytic Nrf2 against neurodegeneration has been suggested by neuroprotective effects of Nrf2 overexpression in astroglial cells in mouse models of amyotrophic lateral sclerosis (ALS) and PD (Chen et al., 2009; Vargas et al., 2008). Moreover, astrocytes have greater antioxidant potential than neurons (Makar et al., 1994; Raps et al., 1989) and many studies also provide evidence for efflux of GSH from astrocytes as a key factor in neuroprotection (Iwata-Ichikawa et al. 1999, Dringen et al. 2000, Dringen and Hirrlinger 2003). Nrf2/ARE activation in astrocytes leading to increased levels of GSH seems to be a major component of the protection conferred by urate.

In addition to the extensive preclinical data supporting a key role of Nrf2 disruption in PD neurodegeneration, recent epidemiological studies have suggested Nrf2 genetic variants modify PD susceptibility and onset (Todorovic et al 2015, Von Otter et al 2010). From a therapeutic standpoint this astrocytic Nrf2-orchestrated defense system may offer an attractive drug target in several neurodegenerative diseases and other neurological disorders. For example, another small molecule dimethyl fumarate (DMF), which has been found effective and approved for use as a disease-modifying treatment of multiple sclerosis, may confer its cytoprotective effects via activation of the Nrf2 pathway (Scannevin et al., 2012). Urate has been gaining momentum as a promising target or agent for neuroprotection based on accumulating epidemiological observations, laboratory data, and encouraging early clinical (including phase II) trial results for several neurological conditions, most actively for PD (Schwarzschild et al., 2014) and stroke (Chamorro et al., 2014). The present findings implicating a discrete astrocytic antioxidant response signaling cascade in the protective actions of urate, in addition to its established but non-specific direct antioxidant properties, strengthen the biological plausibility of its protective potential and support its further clinical development. 


\section{Acknowledgements}

This study was funded by the Department of Defense/NETPR program W81XWH-11-1-0150, NIH K24NS060991, R21 NS084710 and Granite State Development. A visiting fellowship supporting Hong Zhang was funded by National Natural Science Foundation of the People's Republic of China (No: 30572170, 81171886). We thank Michael Maguire for mouse husbandry and cell line maintenance.

\section{Figure Legends}

Figure 1. Marked elevation in GSH release from astrocytes treated with urate. (A) Conditioned medium (CM) from urate-treated astrocytes protects dopaminergic cells from $\mathrm{H}_{2} \mathrm{O}_{2}$ induced cell death. Representative graphs of FACS analysis show cell viability using propidium iodide (PI)/Annexin V staining. Percentages of PI+/AnnexinV+ (dead), PI-/AnnexinV+ (apoptotic) and $\mathrm{PI}-/$ AnnexinV- (vital) staining are shown for untreated MES 23.5 cells, or those treated with CM from vehicle- or urate $(100 \mu \mathrm{M})$-treated astrocytes a day before and during $200 \mu \mathrm{M}$ $\mathrm{H}_{2} \mathrm{O}_{2}$ treatment for $24 \mathrm{~h}$. (B)Targeted screening of several neurotrophic factors in the CM. There was no change detected in levels of BDNF, GDNF and IL6 factors in the urate versus vehicle CM. GSH content was significantly increased in CM from urate- (versus vehicle-) treated astrocyte. *denotes $p$ value $<0.001 ;(n=4$ independent experiments).

Figure 2. Urate induces the Nrf2 pathway in astrocytes.(A) GSH levels are elevated in lysates from $100 \mu \mathrm{M}$ urate- (versus vehicle-) treated astrocytes. (B) Urate induces protein products of Nrf2-targeted genes as shown by western blots of GCLM, GCLC and NQO-1 proteins in urate-treated astrocytes compared to controls. The approximate molecular weight of the indicated protein in $\mathrm{kDa}$ is indicated at Left. The graph represents densitometric analysis of the western blots to semi quantify the protein levels. (C) Quantitative PCR analysis of Nrf2 
target genes in urate- (versus vehicle-) treated cells. Mean \pm SEM are shown $(n=3) .{ }^{*}$ denotes $p$ value $<0.05$

Figure 3. Urate induces nuclear translocation of the Nrf2 protein. (A) Astrocytes were incubated with urate $(100 \mu \mathrm{M})$ or vehicle control for $8 \mathrm{~h}$. Cells were immunostained for astrocytespecific marker GFAP (red) and nuclei were stained with DAPI (blue). Nrf2 was detected using FITC (green) staining. These representative images show a predominant nuclear distribution of the Nrf2 protein after urate treatment, in contrast to the greater proportion of cytoplasmic expression of Nrf2 in vehicle-treated astrocytes. (B) The graph represents the quantification of the nuclear:cytoplasmic (N/C) ratios of Nrf2 staining intensity using ImageJ software. 20-30 cells from 4 independent experiments were counted. * denotes $p$ value $<0.05$.

Figure 4. GSH depletion attenuated the protective effects of urate. (A) GSH content was undetectable in $\mathrm{CM}$ from astrocytes that were treated with urate as well as either BSO $(0.25$ $\mathrm{mM})$ or MK-571 $(50 \mu \mathrm{M})$. (B,C)MES 23.5 cells were exposed to an oxidative stressor $(200 \mu \mathrm{M}$ $\mathrm{H}_{2} \mathrm{O}_{2}$ ) after pretreatment with $\mathrm{CM}$. The $\mathrm{CM}$ was from vechicle- or urate-treated astrocytes, with the latter also treated with or without BSO $(0.25 \mathrm{mM})$ or MK-571 $(50 \mu \mathrm{M})$ as indicated.. The protective effect of CM from urate-treated astrocytes against $\mathrm{H}_{2} \mathrm{O}_{2}$ toxicity (\% dead cells) was significantly reduced by astrocyte incubation with BSO (B) or MK-571 (C) (n=3). * denotes $p$ value $<0.05$ 


\section{References}

Ascherio, A., et al., 2009. Urate as a predictor of the rate of clinical decline in Parkinson disease. Arch Neurol. 66, 1460-8.

Bakshi R, et al., Purines in Parkinson's: Adenosine A2A receptors and urate as targets for neuroprotection. . In: M. Morelli, (Ed.), The Adenosinergic system - a non-dopaminergic target

in Parkinson's disease. Springer, 2015.

Behbahani, H., et al., 2005. Flow cytometry as a method for studying effects of stressors on primary rat neurons. J Neurosci Res. 82, 432-41.

Belanger, M., Magistretti, P. J., 2009. The role of astroglia in neuroprotection. Dialogues Clin Neurosci. 11, 281-95.

Cai, J., et al., 1997. Differential regulation of gamma-glutamylcysteine synthetase heavy and light subunit gene expression. Biochem J. 326 ( Pt 1), 167-72.

Cai, J., et al., 1995. Hormonal and cell density regulation of hepatic gamma-glutamylcysteine synthetase gene expression. Mol Pharmacol. 48, 212-8.

Chamorro, A., et al., 2014. Safety and efficacy of uric acid in patients with acute stroke (URICOICTUS): a randomised, double-blind phase 2b/3 trial. Lancet Neurol. 13, 453-60.

Chen, P. C., et al., 2009. Nrf2-mediated neuroprotection in the MPTP mouse model of Parkinson's disease: Critical role for the astrocyte. Proc Natl Acad Sci U S A. 106, 2933-8.

Chen, X., et al., 2013. Disrupted and transgenic urate oxidase alter urate and dopaminergic neurodegeneration. Proc Natl Acad Sci U S A. 110, 300-5.

Cipriani, S., et al., 2010. Urate: a novel biomarker of Parkinson's disease risk, diagnosis and prognosis. Biomark Med. 4, 701-12.

Cipriani, S., et al., 2012a. Protection of dopaminergic cells by urate requires its accumulation in astrocytes. J Neurochem. 123, 172-81.

Cipriani, S., et al., 2012b. Urate and its transgenic depletion modulate neuronal vulnerability in a cellular model of Parkinson's disease. PLoS One. 7, e37331.

Crawford, G. D., Jr., et al., 1992. A novel N18TG2 x mesencephalon cell hybrid expresses properties that suggest a dopaminergic cell line of substantia nigra origin. J Neurosci. 12, 33928.

Drake, K. R., et al., 2010. Nucleocytoplasmic distribution and dynamics of the autophagosome marker EGFP-LC3. PLoS One. 5, e9806.

Drew, R., Miners, J. O., 1984. The effects of buthionine sulphoximine (BSO) on glutathione depletion and xenobiotic biotransformation. Biochem Pharmacol. 33, 2989-94. 
Dringen, R., Gutterer JM, Hirrlinger J. 2000. Glutathione metabolism in brain metabolic interaction between astrocytes and neurons in the defense against reactive oxygen species. Eur J Biochem. 267, 4912-6.

Dringen, R., Hirrlinger, J., 2003. Glutathione pathways in the brain. Biol Chem. 384, 505-16.

Dringen, R., et al., 1999. Synthesis of the antioxidant glutathione in neurons: supply by astrocytes of CysGly as precursor for neuronal glutathione. J Neurosci. 19, 562-9.

$\mathrm{Du}, \mathrm{Y}$., et al., 2007. Astroglia-mediated effects of uric acid to protect spinal cord neurons from glutamate toxicity. Glia. 55, 463-72.

Duan, W., et al., 2002. Dietary folate deficiency and elevated homocysteine levels endanger dopaminergic neurons in models of Parkinson's disease. J Neurochem. 80, 101-10.

Franklin, C. C., et al., 2009. Structure, function, and post-translational regulation of the catalytic and modifier subunits of glutamate cysteine ligase. Mol Aspects Med. 30, 86-98.

Gong, L., et al., 2012. Neuroprotection by urate on 6-OHDA-lesioned rat model of Parkinson's disease: linking to Akt/GSK3beta signaling pathway. J Neurochem. 123, 876-85.

Guerreiro, S., et al., 2009. Protection of midbrain dopaminergic neurons by the end-product of purine metabolism uric acid: potentiation by low-level depolarization. J Neurochem. 109, 111828.

Hirrlinger, J., et al., 2002. Glutathione release from cultured brain cells: multidrug resistance protein 1 mediates the release of GSH from rat astroglial cells. J Neurosci Res. 69, 318-26.

Iwata-Ichikawa, E., et al., 1999. Glial cells protect neurons against oxidative stress via transcriptional up-regulation of the glutathione synthesis. J Neurochem. 72, 2334-44.

Jones, D. C., et al., 2000. Dopamine-induced apoptosis is mediated by oxidative stress and Is enhanced by cyanide in differentiated PC12 cells. J Neurochem. 74, 2296-304.

Kensler, T. W., et al., 2007. Cell survival responses to environmental stresses via the Keap1Nrf2-ARE pathway. Annu Rev Pharmacol Toxicol. 47, 89-116.

Kraft, A. D., et al., 2004. Nuclear factor E2-related factor 2-dependent antioxidant response element activation by tert-butylhydroquinone and sulforaphane occurring preferentially in astrocytes conditions neurons against oxidative insult. J Neurosci. 24, 1101-12.

Lu, S. C., 2013. Glutathione synthesis. Biochim Biophys Acta. 1830, 3143-53.

Ma, Q., 2013. Role of nrf2 in oxidative stress and toxicity. Annu Rev Pharmacol Toxicol. 53, 401-26.

Makar, T. K., et al., 1994. Vitamin E, ascorbate, glutathione, glutathione disulfide, and enzymes of glutathione metabolism in cultures of chick astrocytes and neurons: evidence that astrocytes play an important role in antioxidative processes in the brain. J Neurochem. 62, 45-53. 
Maragakis, N. J., Rothstein, J. D., 2006. Mechanisms of Disease: astrocytes in neurodegenerative disease. Nat Clin Pract Neurol. 2, 679-89.

Moellering, D., et al., 1998. Nitric oxide-dependent induction of glutathione synthesis through increased expression of gamma-glutamylcysteine synthetase. Arch Biochem Biophys. 358, 7482.

PSG, et al., 2014. Inosine to increase serum and cerebrospinal fluid urate in Parkinson disease: a randomized clinical trial. JAMA Neurol. 71, 141-50.

Raps, S. P., et al., 1989. Glutathione is present in high concentrations in cultured astrocytes but not in cultured neurons. Brain Res. 493, 398-401.

Scannevin, R. H., et al., 2012. Fumarates promote cytoprotection of central nervous system cells against oxidative stress via the nuclear factor (erythroid-derived 2)-like 2 pathway. J Pharmacol Exp Ther. 341, 274-84.

Scott, G. S., et al., 2005. Uric acid protects against secondary damage after spinal cord injury. Proc Natl Acad Sci U S A. 102, 3483-8.

Shih, A. Y., et al., 2003. Coordinate regulation of glutathione biosynthesis and release by Nrf2expressing glia potently protects neurons from oxidative stress. J Neurosci. 23, 3394-406.

Sidoryk-Wegrzynowicz, M., et al., 2011. Role of astrocytes in brain function and disease. Toxicol Pathol. 39, 115-23.

Todorovic, M., et al., 2015. Comprehensive assessment of genetic sequence variants in the antioxidant 'master regulator' nrf2 in idiopathic Parkinson's disease. PLoS One. 10, e0128030.

Vargas, M. R., et al., 2008. Nrf2 activation in astrocytes protects against neurodegeneration in mouse models of familial amyotrophic lateral sclerosis. J Neurosci. 28, 13574-81.

von Otter, M., et al., 2014. Genetic associations of Nrf2-encoding NFE2L2 variants with Parkinson's disease - a multicenter study. BMC Med Genet. 15, 131.

Yu, Z. F., et al., 1998. Uric acid protects neurons against excitotoxic and metabolic insults in cell culture, and against focal ischemic brain injury in vivo. J Neurosci Res. 53, 613-25.

Zhang, N., et al., 2014. Nrf2 signaling contributes to the neuroprotective effects of urate against 6-OHDA toxicity. PLoS One. 9, e100286.

Zhu, T. G., et al., 2012. Protective effects of urate against 6-OHDA-induced cell injury in PC12 cells through antioxidant action. Neurosci Lett. 506, 175-9. 
A

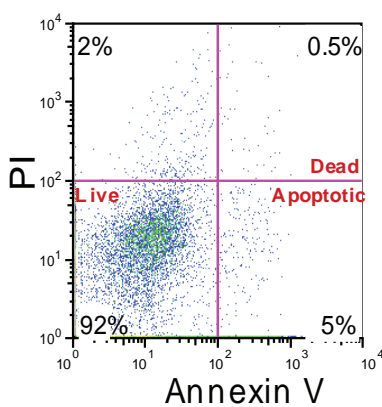

No CM / Untreated

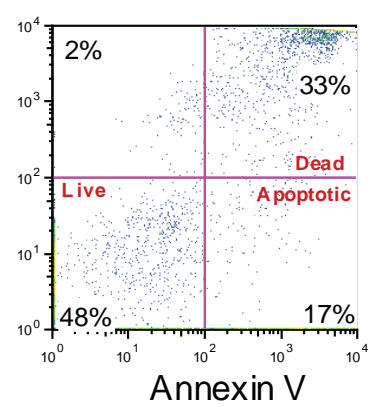

Vehicle $\mathrm{CM} / \mathrm{H}_{2} \mathrm{O}_{2}$

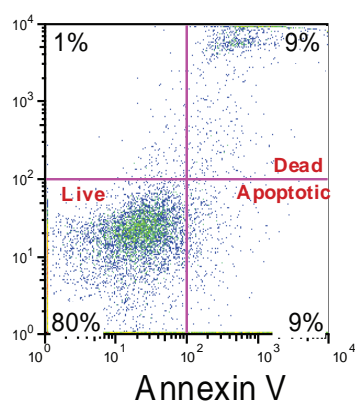

Urate $\mathrm{CM} / \mathrm{H}_{2} \mathrm{O}_{2}$

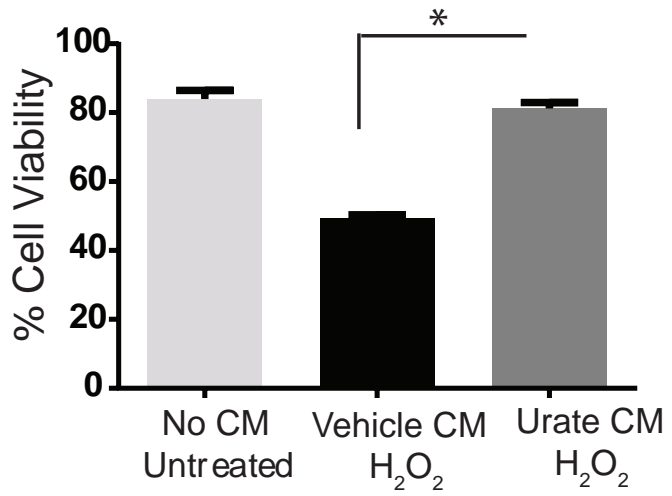

B
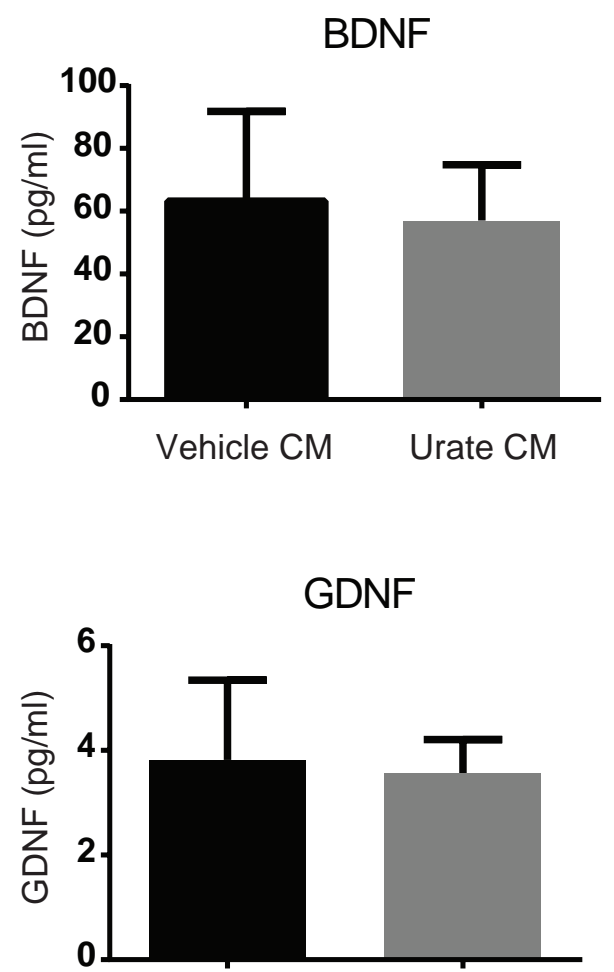

Vehicle CM Urate CM
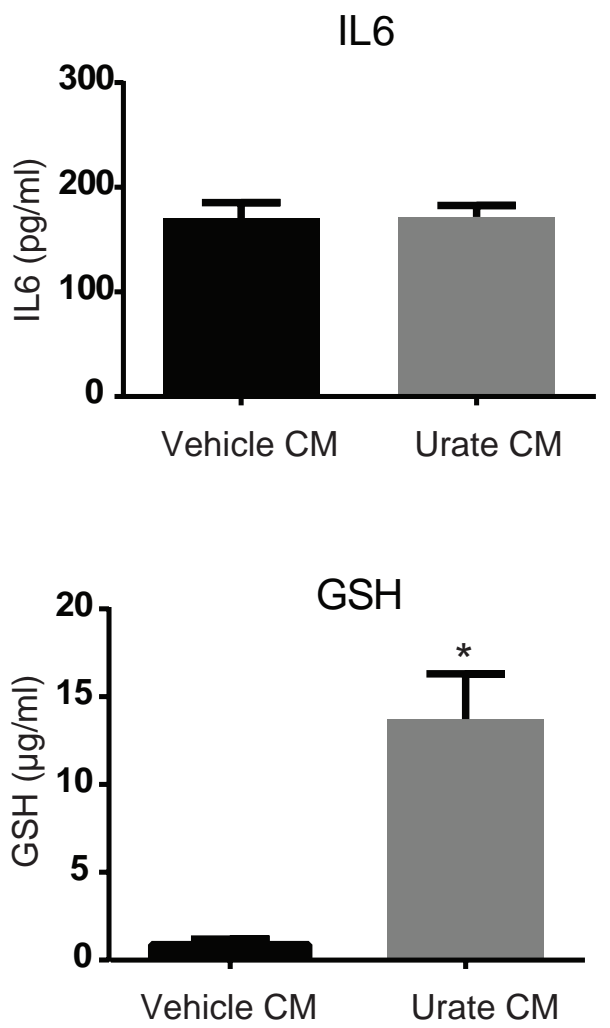
A

B
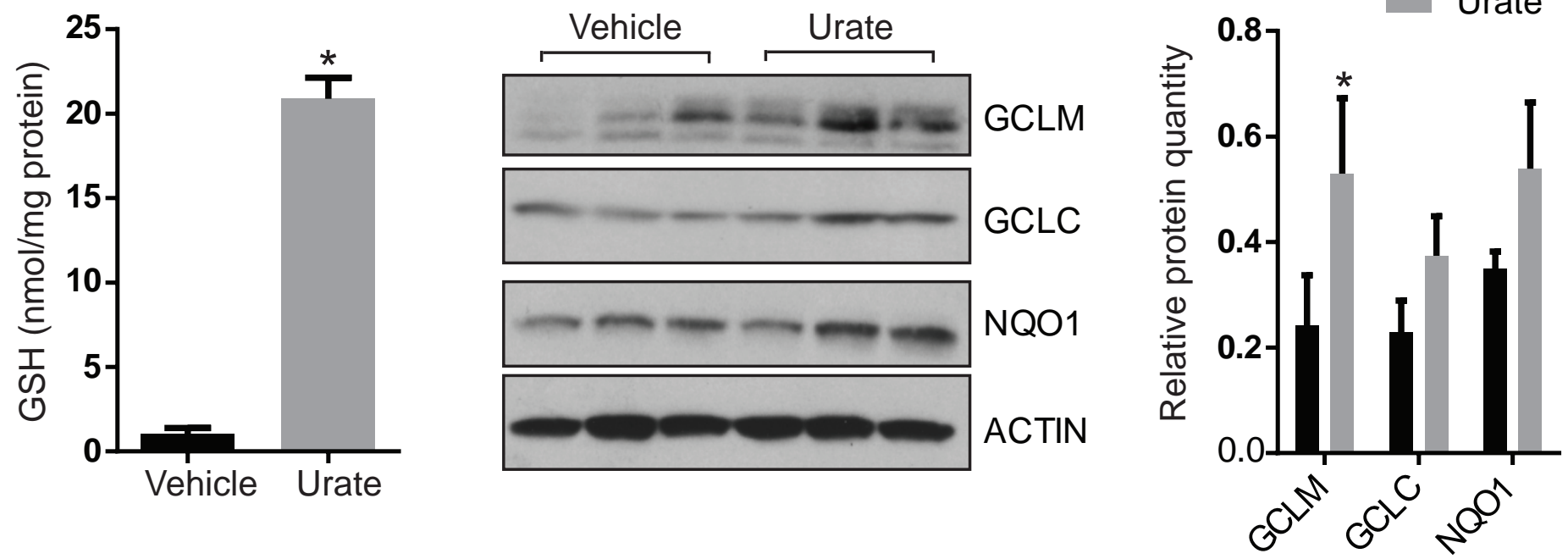

C

GCLM

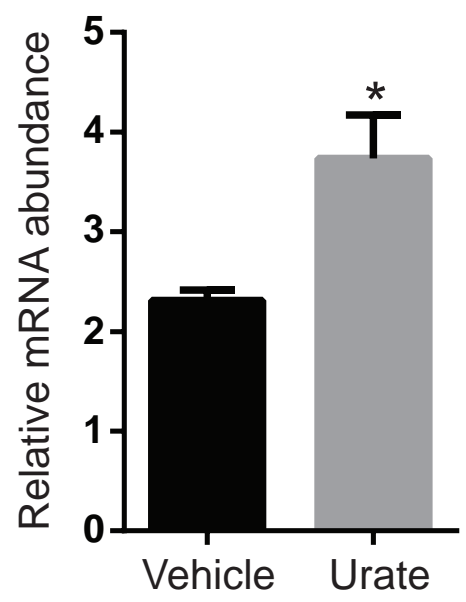

GCLC

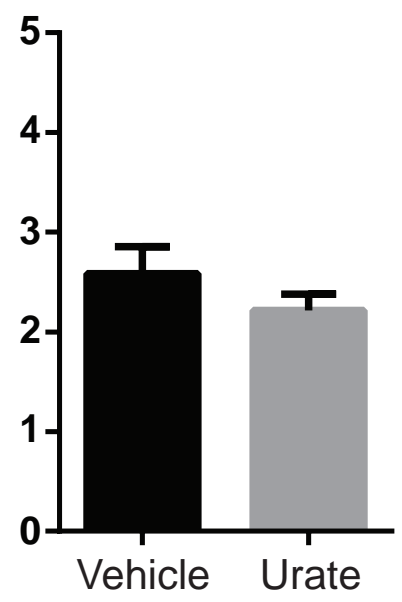

NQO1

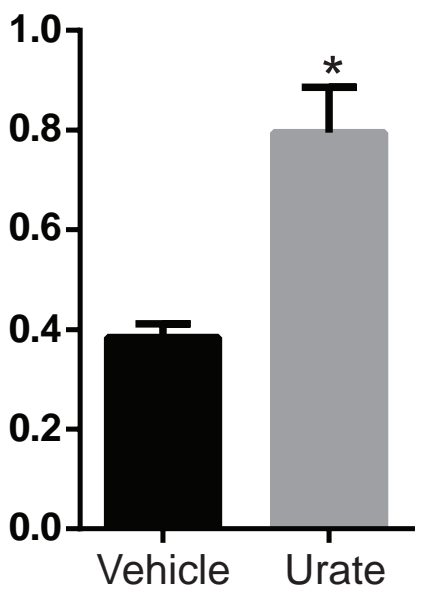

HO-1

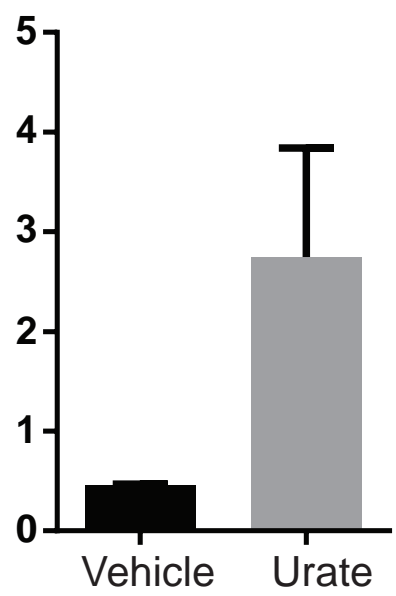


A

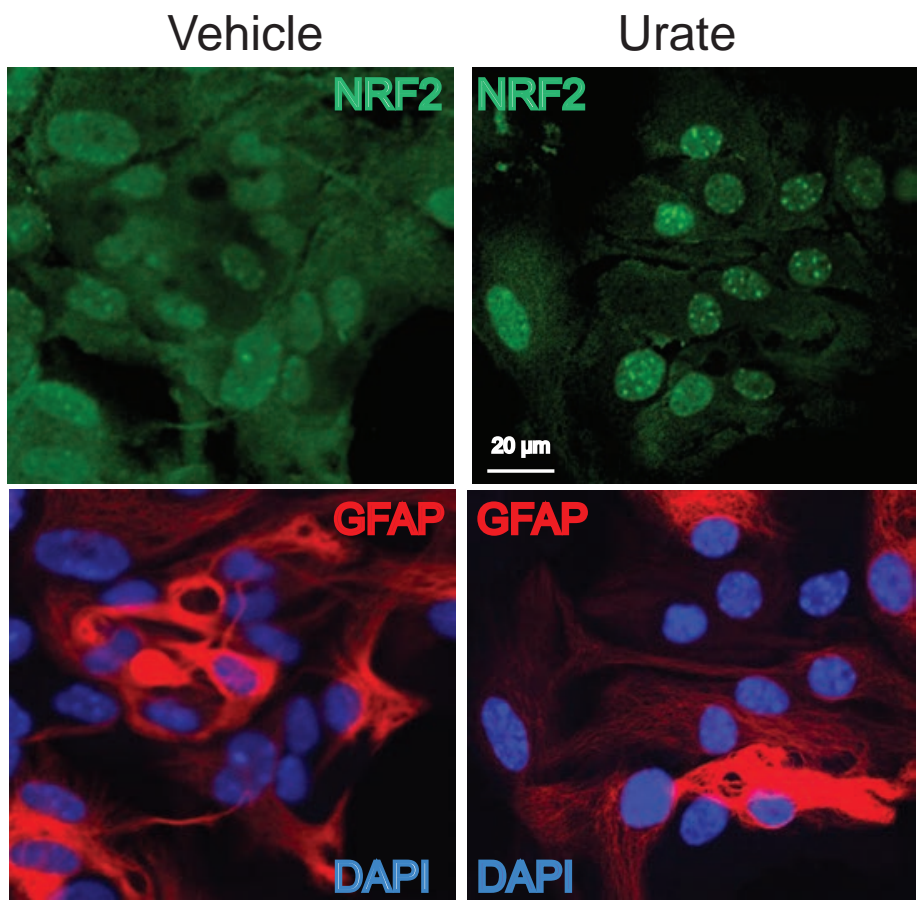

B

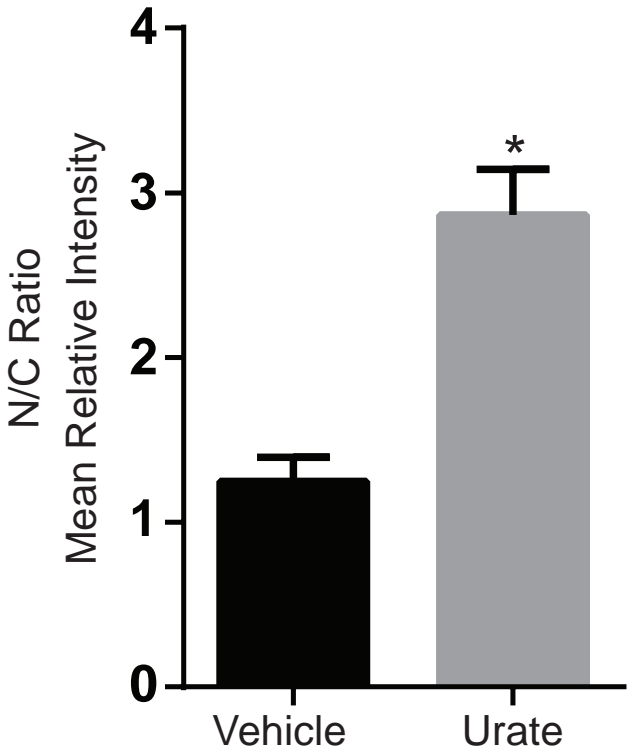



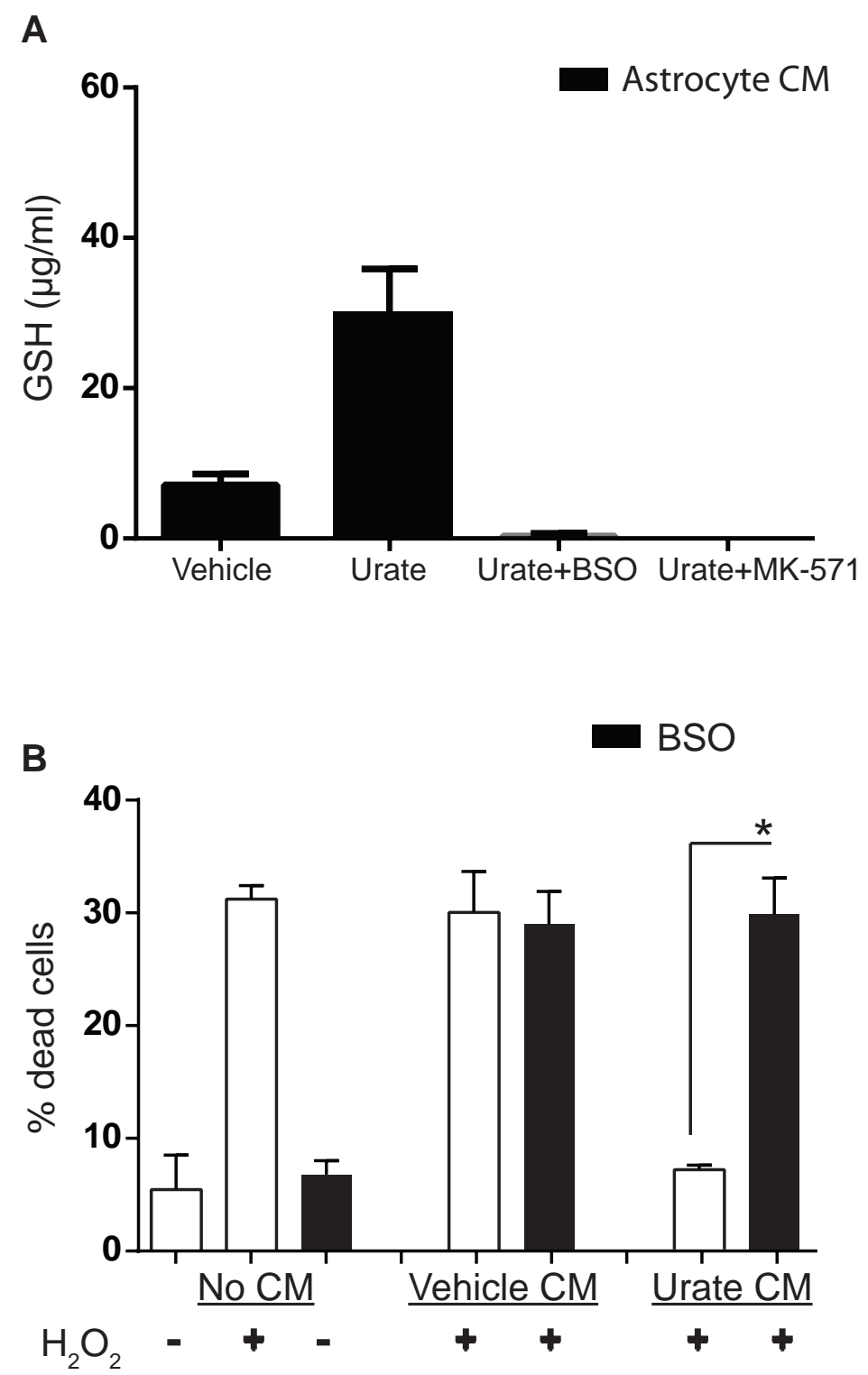

C

MK-571

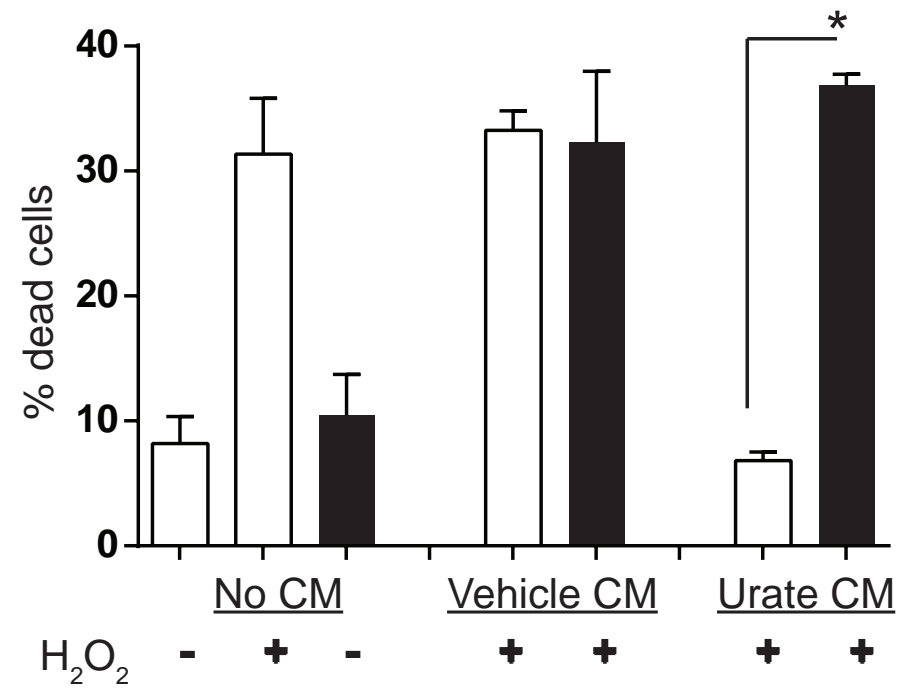

